\title{
HERA DVCS Results
}

\author{
Jonathan Burns ${ }^{* \dagger}$ \\ Glasgow \\ E-mail: j.burnsephysics.gla.ac.uk
}

Deeply Virtual Compton Scattering is the simplest interaction that allows access to Generalised Parton Distributions (GPDs), a theoretical framework that can be used to describe nucleon structure. The strong interest in GPDs results from the fact that they offer access to the total angular momentum of quarks inside the nucleon and to a 3-dimensional picture of nucleon structure. The measurement of the DVCS process is facilitated by a competing interaction known as the BetheHeitler process which has the same final state. GPDs sensitive to asymmetries are accessed from the interference of these two processes. Measurements of DVCS data recorded at H1, ZEUS and HERMES are presented. The cross section results from $\mathrm{H} 1$ and ZEUS are measured as a function of the virtuality of the exchanged photon and the centre-of-mass energy $\mathrm{W}$ of the $\gamma^{*} p$ system. A beam charge asymmetry is extracted for the first time at a collider using H1 data in the low $\mathrm{x} \approx \mathrm{Q}^{2} / \mathrm{W}^{2}$ kinematic domain. A subsample of events from ZEUS is used to produce the first direct measurement of the differential cross section as a function of $t$. Beam charge, beam spin and target asymmetries on both unpolarised and polarised hydrogen and deuterium targets have been extracted at HERMES. The extracted asymmetries are presented over the range of HERMES kinematic acceptance, with their dependence on kinematic variables $\mathrm{t}, \mathrm{x}_{B}$ and $\mathrm{Q}^{2}$ also shown and compared to a phenomenological model.

European Physical Society Europhysics Conference on High Energy Physics

July 16-22, 2009

Krakow, Poland

\footnotetext{
* Speaker.

$\dagger$ On Behalf of the H1, ZEUS and HERMES collaborations
} 


\section{Introduction}

Generalised Parton Distributions (GPDs) [Ji.97] parameterise hard exclusive reactions which leave the target nucleon intact. GPDs are an informational framework in which regular Parton Distributions (PDFs), that describe the longitudinal momentum and helicity distribution of partons, and Form Factors (FFs) that describe the transverse distribution and current density of partons [BD02] are embodied as limiting cases and moments respectively. GPDs also provide information on the total angular momentum carried by quarks in the nucleon. There four nucleon GPDs are $H, \tilde{H}, E$ and $\tilde{E}$. The most experimentally accessible GPD to extract is $H$. Deeply Virtual Compton Scattering (DVCS) is the simplest hard exclusive reaction that can be used to constrain the values of GPDs. DVCS results at low $x_{B}$, measured at H1 and ZEUS, allow the sea quarks of the nucleon to be accessed while the higher $x_{B}$ results at HERMES allow information on the valence quarks to be obtained.

DVCS on the proton $\left(\gamma^{*} p \rightarrow \gamma p\right)$ can be regarded as the scattering of the virtual photon off the proton via a colourless exchange, producing a real photon in the final state. In addition to the DVCS process there is the competing Bethe-Heitler scattering process, which has the same initial and final state as DVCS, resulting from a photon being radiated from the lepton instead of the parton. These two processes are indistinguishable but they do interfere. A number of asymmetry results can be obtained from this interference. The asymmetry results from HERA are dependent on the angle $\phi$, which is defined as the angle between the lepton-nucleon scattering plane and the real photon production plane.

HERA was part of the DESY research centre in Hamburg, Germany. The HERA storage ring consisted of two concentric beams: a $27.5 \mathrm{GeV}$ positron/electron beam and a $920 \mathrm{GeV}$ proton beam. The collider experiments $\mathrm{H} 1$ and ZEUS used both HERA beams while HERMES was a fixed target experiment that used only the electron/positron beam. A lower $x_{B}$ range of $x_{B}<0.01$ was used at $\mathrm{H} 1$ and ZEUS, whilest HERMES explored the range, $0.03<x_{B}<0.35$.

\section{Results}

H1 and ZEUS have similar kinematics hence the data from both experiments is presented together. The cross section measurements are compared to the predictions based on GPD or dipole models [RG02]. These two models are not connected at the present level of understanding, however, at low $\mathrm{x}$ the dipole amplitudes can be used to provide parameterisations for GPDs at a certain scale. The GPD model is sensitive to GPD $H$, based on the partial wave expansions of DVCS amplitudes and represents a first attempt to parametrise all GPDs over the full kinematic domain. The Dipole model used to describe the low x HERA results is based on the gluon density extracted from fits to $F_{2}$ data, the DVCS cross section is computed using a universal dipole amplitude. 


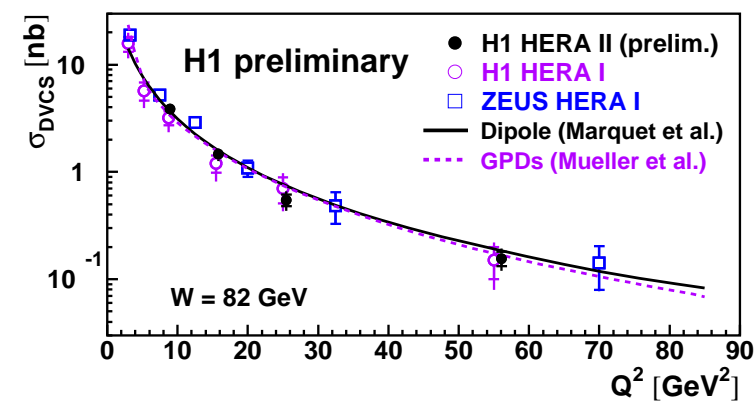

(a) $Q^{2}$

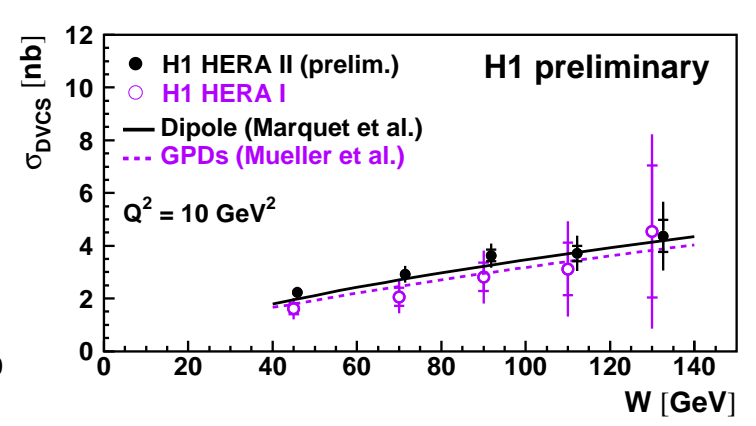

(b) $\mathrm{W}$

Figure 1: The DVCS cross section as a function of $\mathrm{Q}^{2}$ at $\mathrm{W}=82 \mathrm{GeV}$ (a) and as a function of $\mathrm{W}$ at $\mathrm{Q}^{2}=10$ $\mathrm{GeV}^{2}$ (b). The result of predictions from the GPD and Dipole models as well as current and previous results from previous H1 [Col05] and ZEUS publications [eaZC] based on HERA I are also shown.

The cross section shown in Fig 1 decreases with $\mathrm{Q}^{2}$ as expected and the results from both H1 and ZEUS agree with both the GPD and dipole model predictions. The W dependence of the DVCS cross section is determined over a range of $\mathrm{Q}^{2}$. The cross section increases with $\mathrm{W}$ as expected from pQCD-based models. This behaviour is related to the increase of the gluon content of the proton with decreasing $x_{B}$.

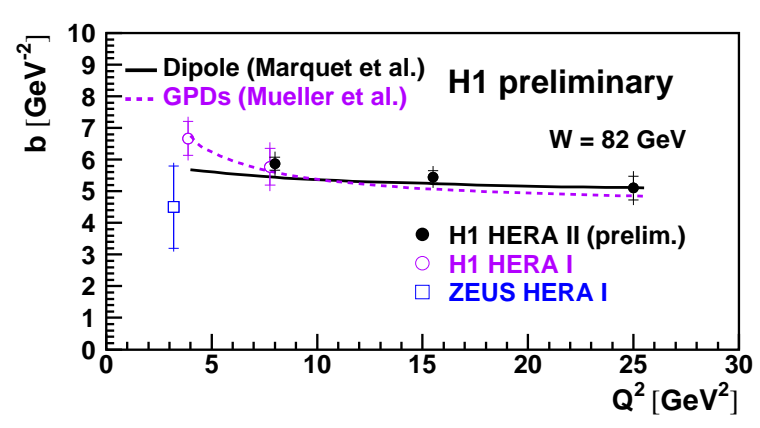

(a) $Q^{2}$

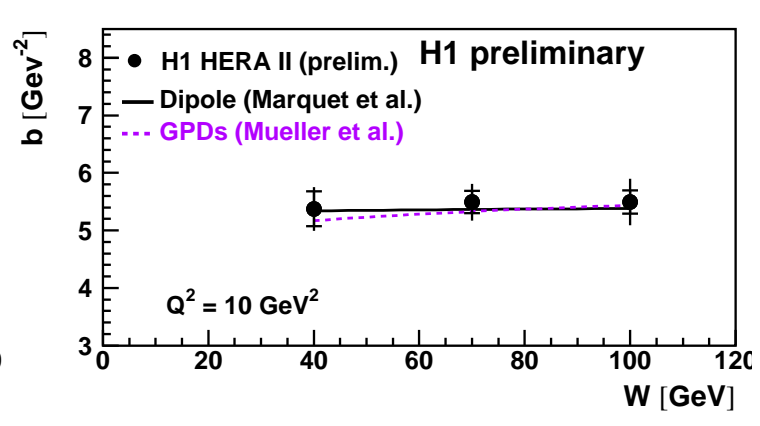

(b) $\mathrm{W}$

Figure 2: The fitted $t$-slope parameters $\mathrm{b}\left(\mathrm{Q}^{2}\right)$ are shown in (a) together with previous $\mathrm{H} 1$ [Col05] and ZEUS [eaZC] results. In (b) the fitted $t$-slope parameters $\mathrm{b}(\mathrm{W})$ are shown.

The DVCS differential cross section extracted from the Leading Proton Scattered (LPS) events at $\mathrm{Q}^{2}=3.2 \mathrm{GeV}^{2}$ and $\mathrm{W}=104 \mathrm{GeV}$ obtained from ZEUS is shown in Fig 2. The differential cross section decreases with |t|. A fit of the form $\mathrm{e}^{-b|t|}$ is made to the data plots resulting in a value of $\mathrm{b}=$ $4.5 \pm 1.3 \pm 0.4$, the former error being statistical and the latter systematic. This value is consistent with the result from $\mathrm{H} 1$ at $\mathrm{Q}^{2}=8 \mathrm{GeV}^{2}$ and $\mathrm{W}=82 \mathrm{GeV}$ giving a value of $\mathrm{b}=5.45 \pm 0.19 \pm 0.34$. The t-dependence of the DVCS cross section allows information on the transverse momentum of protons to be obtained from the data. The derived t-slope parameters are shown with respect to $\mathrm{Q}^{2}$ and $\mathrm{W}$ for inelastic DVCS events. The experimental results from H1 and ZEUS using both HERA running periods are consistent and agree with the results from the GPD and Dipole models. These results show that the value of $\mathrm{b}$ remains constant over the $\mathrm{W}$ range and indicate an increasing value 
of $\mathrm{b}$ at low $\mathrm{Q}^{2}$. The $\mathrm{t}$-slope value can be related to an average impact parameter of $\sqrt{\left\langle r_{T}^{2}\right\rangle}=0.64$ $\pm 0.02 \mathrm{fm}$. This corresponds to the transverse extension of the parton density, which is dominated by sea quarks and gluons at an average value of $x=1.2 \cdot 10^{-3}$, in the plane perpendicular to the direction of motion of the proton.

HERMES was a fixed target experiment that had a higher $\mathrm{x}$ range and a more constrained $\mathrm{Q}^{2}$ range than $\mathrm{H} 1$ and ZEUS. The HERMES collaboration has produced a number of asymmetry results in the azimuthal distribution of the real photon in DVCS [Air01] [Co107]. These include the Beam Spin Asymmetry (BSA), Beam Charge Asymmetry (BCA), Longitudinal Target Spin Asymmetry (LTSA) and Transverse Target Spin Asymmetry (TTSA). H1 has also produced a BCA result for the first time at a collider using equal luminosity of electrons and positrons. The results obtained are compared to model calculations based on a GPD model (VGG) [MV99]. In this model, the t-independent part of the GPD is parameterised by a double-distribution completed by a "D-term". The Beam Charge and Spin asymmetry results have been determined for a number of different amplitudes. The fraction of associated Bethe-Heitler events that cannot be separated from the DVCS events is calculated from a Monte Carlo simulation increases with -t. The Beam Spin Asymmetry shown in Fig 3 amplitudes result from the DVCS interference term. The sin $\phi$ amplitude has a value of approximately -0.2 which is less than predicted by the VGG model and does not display any clear dependence on $-\mathrm{t}, \mathrm{Q}^{2}$ and $\mathrm{x}_{B}$. The $\sin 2 \phi$ is much smaller than the $\sin \phi$ amplitude and is consistent with zero. The other amplitudes are compatable with zero and are included to check the consistency of the extraction method. The VGG model results show a dependence on - $t$ for the $\sin \phi$ amplitude that is not seen in the data. The $\sin \phi$ amplitude is sensitive to the imaginary part of GPD $H$ and from this asymmetry it is possible to constrain a value for $H$ that dominates at the kinematics of the HERA DVCS experiments.

\section{Conclusions}

DVCS interactions at H1, ZEUS and HERMES have been used to investigate and constrain the values of GPDs. The HERA experiments have provided important pioneering results in this exciting and developing field. HERMES data with the Recoil Detector will provide new asymmetry results with the recoiling proton from the DVCS interaction detected directly. GPD $\mathrm{H}$ allows access to the transverse momentum of quarks. Once a value of GPD $H$ has been constrained it will be possible to constrain GPD $\tilde{H}$ from the HERMES TTSA result and further work will allow values for the remaining GPDs, $E$ and $\tilde{E}$, to be completed. Future work at experiments such as CLAS and the continuing work of theorists to constrain GPD models will provide new insights on nucleon structure. 


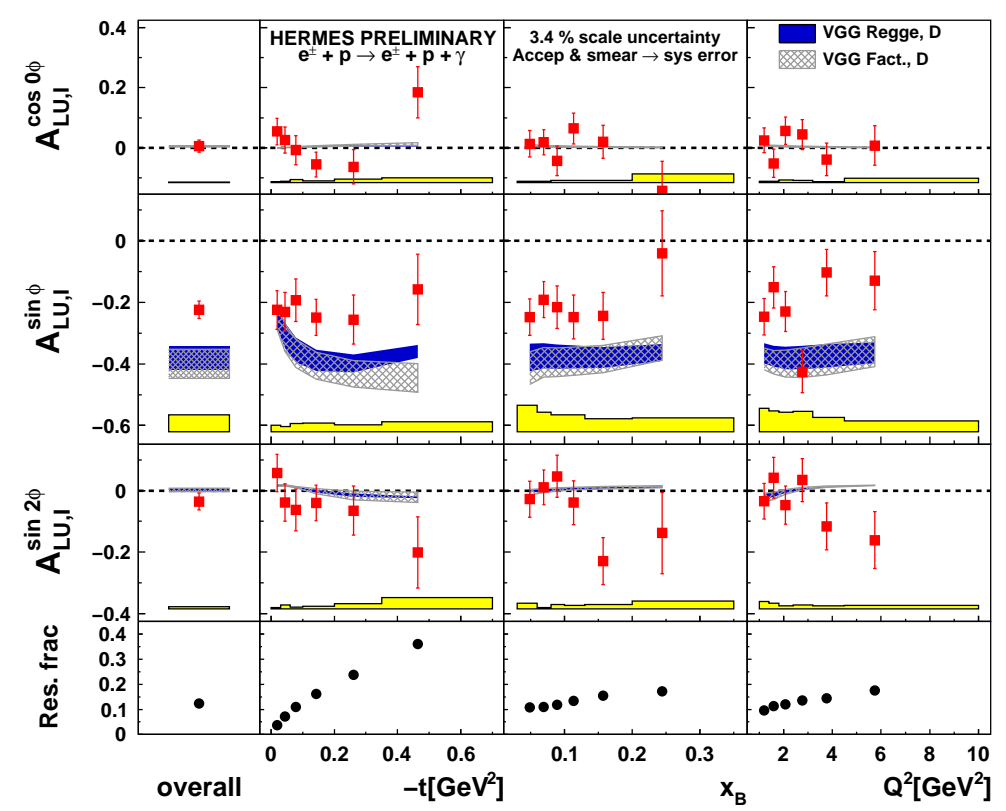

Figure 3: The first row shows the $\cos 0 \phi$ amplitude of the beam spin asymmetry $\mathrm{A}_{L U}$. The second and third rows show the $\sin \phi$ and $\sin 2 \phi$ amplitude terms accordingly that are sensitive to the interference term. The amplitudes are measured on a hydrogen target from $1996-2005$ as a function of $-\mathrm{t}, \mathrm{Q}^{2}$ and $\mathrm{x}_{B}$. The VGG model curves are displayed as is the systematic error band. The bottom row shows the fractional contribution of associated Bethe-Heitler production as obtained from a Monte Carlo simulation.

\section{References}

[Air01] HERMES Collaboration: A. Airapetian. Measurement of the beam-spin azimuthal asymmetry associated with deeply-virtual compton scattering. Phys.Rev.Lett., 87:182001,2001, Phys.Rev.Lett.87:182001,2001.

[BD02] A.V. Belitsky and D.Muller. Nucleon hologram with exclusive leptoproduction. Nucl. Phys., A711:118, 2002.

[Col05] A.Aktas [H1 Collaboration]. Measurement of deepy compton scattering at hera. Eur. Phys. J. C, 44, 2005.

[Col07] The HERMES Collaboration. The beam-charge azimuthal asymmetry and deeply virtual compton scattering. Phys.Rev.D, 75:011103,2007, Phys.Rev.D75:011103,2007.

[eaZC] S.Chekanov et al. [ZEUS Collaboration]. A measurement of the $\mathrm{q}^{2}, \mathrm{w}$ and $\mathrm{t}$ dependences of deeply virtual compton scattering at hera. JHEP.

[Ji.97] X. Ji. Gauge-invariant decomposition of nucleon spin and its spin-off. Phys. Rev.Lett., 78:610, 1997.

[MV99] M.Guidal M. Vanderhaeghen, P.A.M. Guichon. Deeply virtual electroproduction of photons and mesons on the nucleon : Leading order amplitudes and power corrections. Phys. Rev. D., D60, 1999.

[RG02] M. McDermott R.Sandapen and G.Shaw. Colour dipoles and virtual compton scattering. Eur. Phys. J. C, 22:655, 2002. 